Conclusion Results have shown significant down regulation of FABP4 and PPARG in 3T3-L1 adipocytes induced by HCT 116 colon cancer cells. Rosiglitazone, a potent PPARG agonist, could not rescue expression of FABP4 in 3T3-L1 co-cultured adipocytes. These results provide evidence of colon cancer cell induced adipocyte de-differentiation through PPARG antagonism, which may have a role in sustaining cancer cell survival and progression.

\section{PWE-058 COLONOSCOPY SURVEILLANCE FOR ADENOMATOUS POLYPS: ARE WE DOING IT RIGHT! A RETROSPECTIVE AUDIT}

Imran Tahir*, Ben Hicken, Danny Cheung. Worcestershire Acute Hospitals NHS trust, Worcester, UK

\subsection{6/gutjnl-2019-BSGAbstracts.382}

Introduction Colorectal cancer (CRC) is the third most commonly diagnosed cancer worldwide. The identification of colonic polyps can reduce CRC mortality through earlier diagnosis and removal of polyps: the precursor lesion of CRC. Following the initial colonoscopy, finding and removal of a polyp, some patients are at increased risk of developing CRC in the future. This is the rationale for post-polypectomy surveillance colonoscopy. Not all polyps seen are adenomas with potential of malignant transformation. The potential benefits of surveillance procedures must be weighed against the burden of colonoscopy: resource use, the potential for patient discomfort, and the risk of complications. The aim was to assess the practice for polyp surveillance against gold standard BSG guidelines based on polyp number, size and histological features.

Methods A retrospective audit of colonoscopies performed in Worcestershire Acute Hospitals NHS Trust in 2017 where polyps were reported. Patients were excluded if cancer was identified, a duplicate procedure performed or they were on another surveillance programme. Colonoscopy reports, histology and clinic letters were reviewed to assess planned followup and compliance.

Results 1378 colonoscopies reported polyps. 260 patients were excluded. 1118 patient notes were reviewed. 674 men (60\%) and 444 females (40\%) were assessed with an average age of 67. $965(86 \%)$ were compliant with BSG guidelines. 153 (14\%) patients were not compliant. Of those not compliant, $16(10 \%)$ should have been screened more frequently and $102(67 \%)$ should have received less frequent colonoscopy follow-up. $35(23 \%)$ patients did not require any follow-up as histology did not demonstrate an adenoma incurring a cost implication of $£ 16,275$ to the trust $(£ 465$ tariff per procedure).

Conclusions The results provide insight into the importance of appropriate surveillance. It highlights the implications of unnecessary procedures in terms of costs, psychological stress and risks associated with the procedure. Of those not requiring follow-up it was apparent the endoscopist was predicting the follow-up timescale prior to receiving the histology report, commonly mis-identifying hyperplastic polyps as adenomas. The predicted size of the polyp also varied between endoscopist and histologist. Keeping a record of surveillance patients and filtering out un-necessary procedures may reduce this risk.
PWE-059 QFIT IS A VALUABLE TRIAGE TOOL FOR SYMPTOMATIC COLORECTAL PATIENTS

Michelle Thornton*, Laura Herron, Ian Godber, Martin Downey, Hakim Benyounes. Nhs/, Wishaw, UK

\subsection{6/gutjnl-2019-BSGAbstracts.383}

Introduction To prospectively assess the predictive value of qFIT in symptomatic colorectal patients

Methods Following a pilot in 2013, all symptomatic colorectal patients referred to secondary care were required to provide a stool sample for qFIT testing. A qFIT result $>9.9$ was deemed positive. Patients were clinically reviewed or investigated dependent on symptoms and fitness. A prospective database allowed for electronic case note and GP record review of all patients in December 2018. The database was cross-referenced with the colorectal cancer database.

Results Between July 2013 and September 2018, 4425 patients were tested, 2891 negative(65\%). Data is provided on all patients with complete colorectal investigations or no investigations but greater than 6 months validated clinical follow-up. Of the 2443 negative patients(85\%) with complete data, 16 colorectal cancers (crc), 11 high risk adenomas(HRA), 40 inflammatory diagnoses (IBD) and 151 low risk adenomas (LRA) were diagnosed. The cumulative NPVs were $99.3 \%$, 98.9\%, 97.3\% and $91.1 \%$ respectively.

Of the 1477 positive patients (96\%) with complete data, 99 crcs, 22 HRAs, 66 IBD and 202 LRA were diagnosed. The cumulative PPVs were 6.7\%, 8.2\%, $12.7 \%$ and $26.3 \%$ respectively. In those patients with a qFIT >399 ( $\mathrm{n}=396) 51 \mathrm{crcs}$, 6 HRA, 19 IBD and 45 LRA were diagnosed, giving cumulative PPVs of $12.9 \%, 14.4 \%$, and $30.6 \%$.

Conclusions qFIT is a valuable tool to safely triage patients. The result allows for priority access to resource limit ed investigations and can help avoid risk in patients who test negative.

\section{PWE-060 CT COLONOGRAMS IN ELDERLY PATIENTS, A SAFE AND ACCURATE COLONIC EXAMINATION}

Neel Raja, Michael Adeleye, Bhavini Billimoria, Ajay Verma*. Kettering General Hospital NHS FT, UK

\subsection{6/gutjnl-2019-BSGAbstracts.384}

Introduction In Kettering General Hospital (KGH) we perform almost 500 CT colonograms (CTCs) annually for patients who; do not want, are unable to tolerate, or have failed a colonoscopy (which is considered the "gold standard" colonic examination). The most common reasons for CTC are iron deficiency anaemia, and change in bowel habit, often with the aim of detecting colorectal cancer (CRC).

We have seen an increase over time, in the use of CTC in elderly patients, as a first line investigation. With an ageing population, we explore the significance of performing this examination, in an elderly population who may not be suitable for further investigation/intervention.

Methods We reviewed 1479 patients who had undergone a CT Colonogram between October 2015 and October 2018. Of these, we focused on patients aged $\geq 80$ at the time of scanning. CTC reports were analysed and categorised into those with positive, indeterminate findings, and those with no significant findings. All patients $\geq 80$ years old were followed up (via their electronic records) to observe their outcomes. 